\section{Feline acromegaly: what options should we be discussing with clients?}

\section{Robert Shiel}

Hypersomatotropism is most common in middle-aged to older male cats. Traditionally, clinical signs reported by owners included weight gain, lameness, neurological signs, enlargement of the paws, broad facial features, increased upper respiratory noise and signs associated with poorly controlled diabetes mellitus. In early reports, insulin-resistant diabetes mellitus was also described as a consistent feature. However, it is now recognized that cats may have few or no physical changes suggestive of the disease, and diabetes mellitus may not always be associated with insulin resistance. Rarely, hypersomatotropism may occur in the absence of diabetes mellitus. There are no routine clinicopathological changes that are suggestive of hypersomatotropism in cats. Changes associated with diabetes mellitus are expected.

The measurement of serum insulin-like growth factor (IGF)-1 concentration is the most commonly used method for the diagnosis of hypersomatotropism in cats. Assay performance is variable, and it is essential that any assay is validated specifically for use in the cat. The positive predictive value of serum IGF-1 concentrations greater than $1000 \mathrm{ng} / \mathrm{ml}$ is high. The sensitivity and optimal cut-off of the test are not known; however, acromegaly should not be excluded if serum IGF-1 concentrations are within the upper half of the reference interval, particularly if suspicious clinical signs are present. Serum IGF-1 concentrations should not be measured at the time of diagnosis of diabetes mellitus. Portal insulin is necessary for IGF-1 production. Measurement of IGF-1 concentrations is typically not performed until after 4-8 weeks of insulin administration. Computed tomography or magnetic resonance imaging of the brain may be used to confirm the presence of a pituitary mass.

There are three primary methods of treating feline hypersomatotropism: radiation therapy, hypophysectomy and medical therapy. A good clinical response is seen in most cases following radiation therapy, characterized by decreased insulin resistance and improved glycaemic control. Diabetic remission can occur in some cases. Serum IGF-1 concentrations often remain elevated, and features related to soft tissue and skeletal overgrowth persist. Unfortunately, not all cats respond, and in some cases the response is not sustained.

Surgery is the treatment of choice in human medicine and is being offered at a growing number of veterinary referral centres. Successful treatment has been reported, with normalization of both growth hormone and IGF-1 concentrations, as well as resolution of the diabetic state. However, temporary administration of DDAVP, and lifelong administration of cortisone and levothyroxine, are necessary. Limited medical options are available. A long-acting formulation of pasireotide has shown promise in a small case series. Due to the high cost of surgery and radiation, and poor response to medical therapy, it is not uncommon that acromegaly is left untreated. In such cases, a prolonged response to treatment can be observed despite the requirement to use high doses of insulin. In such cases, remission is unlikely.

\section{KEY LEARNING OBJECTIVES}

- Recognize the cases in which feline hypersomatotropism should be suspected

- Understand interpretation of diagnostic tests for this disease

- Be able to discuss treatment options confidently with clients

\section{MULTIPLE CHOICE QUESTIONS}

1. What treatment for hypersomatotropism is most likely to reduce IGF-1 and growth hormone concentrations in cats with hypersomatotropism?

(A) Hypophysectomy

(B) Pasireotide therapy

(C) Pituitary radiation therapy

(D) Cabergoline therapy

2. Which of the following clinical signs is most suggestive of hypersomatotropism in a cat with diabetes mellitus?

(A) Recurring urinary tract infections

(B) Severe peripheral neuropathy

(C) Weight gain despite poor diabetic control

(D) The presence of ketoacidosis

3. What hormones require long-term replacement in most cats following hypophysectomy?

(A) Cortisol and thyroid hormones

(B) Vasopressin and thyroid hormones

(C) Aldosterone and cortisol

(D) Growth hormone and vasopressin 